\title{
EL EYÁMIKEKWA Y EL AYAHUASQUERO: Las Dinámicas SOCIOECOlÓGICAS del Chamanismo Ese Eja
}

\section{Miguel N. Alexiades}

El presente articulo describe dos elementos del chamanismo Ese Eja. El primero está asociado al eyámikekwa, identificado por los Ese Eja como el curandero tradicional. Un segundo complejo de prácticas chamánicas se basa en el empleo del alucinógeno vegetal ayahuasca. Introducido a comienzos de siglo, el ayahuasca está estrechamente asociado al contacto con mestizos y otras etnias amazónicas, agrupados bajo la categoría deja. A diferencia del eyámikekwa, los rituales y discurso del ayahuasca hacen multiples referencias a deja, a diversos objetos manufacturados, así como a árboles y a ciertos animales acuáticos. El significado de estos modelos chamánicos y sus interacciones son examinados en el contexto de cambio social y ecológico asociado a la intensificación de las relaciones entre los Ese Eja y la sociedad nacional.

This article describes two distinct, though not necessarily mutually exclusive, aspects of Ese Eja shamanism. The first is associated with the eyámikekwa, a ritual specialist identified by the Ese Eja as the "traditional" healer. A second form of shamanistic practice centers on the use of the hallucinogenic beverage ayahuasca. Introduced earlier this century, ayahuasca is explicitly associated by the Ese Eja as a result of contact with non-Ese Eja, subsumed under the category of deja. In sharp contrast to eyámikekwa shamanism, ayahuasca discourse and rituals make multiple references to deja, manufactured objects, trees and to certain aquatic animals. Moreover, the meaning and interaction between shamanistic discourses can be productively examined in the context of social and ecological change, following intensified contact with agents of the nation-state earlier this century. 


\section{INTRODUCCIÓN}

Los trabajos de Reichel-Dolmatoff (1971), Dole (1964), y posteriormente de Brown (1988) y Chaumeil (1983) entre otros, han resaltado las múltiples facetas del chamán como agente mediador de relaciones sociales y ecológicas. Si bien los trabajos de Harner (1984) y Whitten (1976) examinan el chamanismo en el ámbito de relaciones interétnicas y del contacto con la sociedad nacional, los ensayos de Taussig (1987), y mas recientemente de Gow (1994) y Albert (1993) sugieren que el chamanismo amazónico incorpora y refleja una compleja trayectoria de relaciones coloniales y neocoloniales.

Entre los Ese Eja', el chamanismo se mantiene como institución dinámica, compleja y variable. Los Ese Eja reconocen dos modalidades de conocimiento o práctica chamánica, los eyámikekwa o curanderos "tradicionales"2, y el ayahuasca- preparación vegetal alucinógena recientemente incorporada en el sistema medico y ritual Ese Eja. Espero demostrar cómo las características y relación entre estas dos modalidades de practica chamánica reflejan diferentes aspectos de la realidad histórica, social y ecológica de los Ese Eja. Aun más importante, las circunstancias geográficas e históricas del contacto entre los Ese Eja y la sociedad nacional han resultado en la sobreposición e interrelación de varios procesos sociales y ecológicos, como son el colapso demográfico, la migración hacia los cursos bajos de los ríos, y la reestructuración de las relaciones sociales y ecológicas. Sugiero que estos procesos son incorporados y manipulados simbólicamente

Los Ese Eja son un grupo de habla Tacana distribuido a lo largo del ríos Tambopata, Heath, Madre de Dios y Beni, en zonas colindantes de la Amazonia Peruana y Boliviana. El presente ensayo se basa en 20 meses de trabajo de campo en cuatro comunidades Ese Eja del Río Heath y Beni, durante los años 1994-1997, así como en múltiples visitas a otra comunidad en el río Tambopata, durante los últimos 10 años. Mi exposición se limita a una sola comunidad del Río Heath, ya que esta es la única comunidad donde actualmente hay un eyámikekwa, curandero Ese Eja.

2 La designación de tradicional corresponde a una apreciación Ese Eja, que identifica a este tipo de chamanismo con prácticas ancestrales. En este caso, "tradicional" vendría a significar todo aquello relacionado a "los antiguos" (etiikiana), y a un pasado mítico-histórico. 
por el idioma chamánico, articulando la sociedad Ese Eja a una nueva realidad socioecológica y dinámica.

\section{EL EYÁMIKEKWWA}

La palabra eyámikekwa está compuesta por un sustantivo y un verbo. El morfema [eyami] ${ }^{3}$ se refiere al cuerpo físico y tangible de personas y animales, mientras que el verbo transitivo [kekwa] tiene múltiples significados relacionados al acto de penetrar o perforar con un objeto punzante, como por ejemplo una flecha, arpón o estaca. De manera general, [kekwa] también significa cazar, "chontear" "y por ende, matar. Las flechas invisibles con las cuales los edósikiana enferman y matan a los humanos, aparecen, en manos del eyámikekwa, como astillas de chonta (Bactris sp., Iriartea sp.) o paca (Guadua sp.). Esta transformación entre lo ordinariamente invisible y lo visible, personifica el papel del eyámikekwa como intermediario entre los edósikiana y los Ese Eja. La relación entre el eyámikekwa y los edósikiana es compleja, ambivalente y frágil ${ }^{6}$. Si bien algunos edósikiana "chontean" a los Ese Eja para comérselos”, otros los “chontean" para convertir a los Ese Eja en eyámikekwa. El eyámikekwa deriva su poder de los edósikiana, y su habilidad para curar ciertos tipos de enfermedades, prevenir los desastres naturales y las

Para los Ese Eja, el cuerpo, incluyendo los órganos y las excreciones, son manifestaciones o proyecciones de eshawa, la fuerza vital y indestructible de todos los seres.

El verbo chontear se usa para describir la acción por parte de los edósikiana, de disparar a las personas con sus flechas invisibles.

Los edósikiana son seres sobrehumanos que personifican las fuerzas productivas y destructivas de la naturaleza, y del bosque específicamente.

El sufijo-kiana es un indicativo de plural. Si embargo, en sus narrativas, los Ese Eja invariablemente se refieren a "el edósikiana". Chavarría y Sánchez (1991) sugieren que la traducción al singular refleja una influencia misionera monoteísta. Si bien esto puede ser un factor importante, Peluso (en preparación) sugiere que la ambivalencia y multiplicidad son aspectos importante de los agentes sobrenaturales y míticos del universo Ese Eja. A lo largo del texto, empleo ambas formas.

Para los Ese Eja, la enfermedad y la muerte son manifestaciones visibles del acto de predación de los edósikiana sobre los humanos. 
epidemias y garantizar la abundante caza dependen enteramente de la relación de reciprocidad que mantiene con estos, o al menos con algunos (cf. Chavarría, 1996; Kimura, 1981). Como dicen los Ese Eja, "el eyámikekwa cura mediante el edósikiana, madre del monte".

El eyámikekwa sirve de mediador en otros contextos. Así por ejemplo, el eyámikekwa es el vehículo a través del cual los antepasados muertos, los emánokwana, visitan a los vivos. Los rituales comunales eshasha-poi, epowi-sese, y emánokwana tienen como fin mantener viables las relaciones sociales entre los vivos y los muertos, los Ese Eja y los edósikiana. Dichas relaciones también se basan en actos de reciprocidad; los Ese Eja convidan a los emánokwana y al edósikiana a tomar chicha de plátano (epowi-sese o eshasha-poi) y estos a su vez curan a los Ese Eja, les previenen sobre posibles amenazas por parte de ciertos espíritus o fuerzas malignas de la naturaleza y les avisan sobre la ubicación de las manadas de huanganas, $\tilde{n} o^{8}$ (ver Chavarría, este volúmen). Las huanganas son otra manifestación física de los muertos entre los vivos ya que estos animales son para los Ese Eja, como para otros muchos grupos amazónicos (ej. Pollock, 1992 entre los Culina), los muertos que vienen a visitar el mundo de los vivos, "para comer frutas del monte". El eyámikekwa es, por tanto, el medio a través del cual los Ese Eja establecen y mantienen relaciones críticas de intercambio con los distintos "espacios" del universo, y en particular con los animales de caza y los antepasados. Estas relaciones garantizan a la vez el bienestar a través de la caza y la falta de agresiones por parte de agentes sobrehumanos (enfermedades). De allí el comentario de un anciano que " los antepasados siempre hacían eshasha-poi...por eso los niños estaban gordos [sanos]..."

Así como señala Chaumeil (1985), la guerra y predación también constituyen, dentro de la cosmovisión indígena, formas de intercambio recíproco que expresan la interdependencia entre los distintos seres del

Las huanganas, (Tayassu pecari), son una especie de cerdo salvaje que vive en manadas nómadas de hasta varios centenares de individuos. Esta especie, muy preciada por su carne entre los Ese Eja, era de suma importancia dentro de la subsistencia Ese Eja hasta hace poco. 
universo. Al igual que los Ese Eja matan a los animales con sus flechas (o más recientemente con armas de fuego), los edósikiana matan a los Ese Eja con las suyas. La importancia simbólica de la caza como modelo de intercambio en el lenguaje chamánico de los eyámikekwa es consistente con el rol central de ésta dentro de la subsistencia y cultura Ese Eja.

Los Ese Eja frecuentemente lamentan la desaparición de los eyámikekwa, ya que estos eran los únicos que pueden extraer la chonta de los edósikiana. Al no haber mas eyámikekwa se corta un vínculo crítico con los edósikiana y con ciertos "espacios" de la naturaleza y del universo. Por tomar un ejemplo, actualmente la mayor parte de los Ese Eja no pueden hablar con los emánokwana, los muertos9. La observación que ya no se observan las manadas de huanganas de antaño es interpretada por los Ese Eja como síntoma de una ruptura en las relaciones "sociales" con el edósikiana y con los muertos. La quiebra de las relaciones con el edósikiana es también peligrosa, ya que al no ser "alimentado" por los Ese Eja durante las tomas de eshasha-poi, el edósikiana busca alimentarse de los Ese Eja (Burr, 1997: 220).

Si bien el chamanismo eyámikekwa fue tiempo atrás parte de una institución social extendida, su importancia comienza a decrecer aproximadamente partir de los años 30, o sea, paralelamente a la inserción de los Ese Eja dentro de la sociedad nacional ${ }^{10}$. Mientras que hace 50 años hasta uno de cada tres hombres adultos eran eyámikekwa, hoy día solamente queda un eyámikekwa en todo el grupo Ese Eja. La desaparición de los

9. Esta visión contrasta, superficialmente al menos, con la de los conservacionistas. El discurso que las huanganas están desapareciendo debido a la caza desmesurada no concuerda con la cosmovisión Ese Eja, que brinda a la naturaleza, y a los eshawa en particular de japanakiani, la capacidad de replicarse o reproducirse indefinidamente. Analizando estos discursos mas profundamente, sugiero que ambos diagnósticos expresan diferentes aspectos de un mismo proceso y que, por tanto, comparten elementos comunes (Alexiades, en preparación). externos siglos antes, estos contactos fueron, por lo general, limitados tanto en su escala como en su duración. Las últimas décadas del siglo XIX y, sobre todo, las primeras del siglo XX marcan el comienzo de la integración general de los Ese Eja en la sociedad nacional. 
eyámikekwa es atribuida por los Ese Eja a las epidemias de antaño, así como a la "desgana" de los jóvenes y su inhabilidad de "aguantar el chonteado del edósikiana". Si bien el primer factor atribuye la desaparición de los eyámikekwa directamente al contacto con la sociedad nacional, el segundo factor sugiere un cambio en la disposición o habilidad de los más jóvenes, los cuales no pueden "aguantar" el dolor físico y el sufrimiento como lo hacían sus ancestros. El ayahuasca es considerado en este contexto como una alternativa parcial y mas accesible que el poder adquirido mediante el chonteado del edósikiana. Ciertamente, el declive del chamanismo eyámikekwa coincide con la incorporación del ayahuasca en la sociedad Ese Eja.

\section{EL AYAHUASCA ${ }^{11}$}

Las primeras experiencias con ayahuasca ${ }^{12}$, jono, entre los Ese Eja del río Heath se remontan a los años 40 , cuando, siguiendo el cierre de la misión de Lago Valencia, estos comienzan a asentarse en "barracas" junto a patrones, ribereños y otros actores sociales agrupados bajo la categoría deja ${ }^{13}$. Este periodo en la historia del grupo marca la continuación de un periodo de reorganización y reestructuración social y ecológica, evidenciado por el

11 Mi caracterización del ayahuasca se limita a los Ese Eja del río Heath. En el río Tambopata, el uso del ayahuasca está fuertemente influenciado por la presencia reciente de varios curanderos Shipibo-Conibo y Ribereños, y por lo cual difiere del patrón hallado en otras comunidades en varios aspectos.

El nombre ayahuasca se refiere a la planta Banisteriopsis caapi Griseb. (Morton), Malpighiaceae, así como al preparado alucinógeno preparado con esta y otros aditivos. Entre los Ese Eja, el aditivo principal son las hojas de chacruna, Psychotria viridis R. \& P., Rubiaceae.

13 Como etiqueta de identidad social, la categoría de deja es plástica y compleja. Básicamente deja son todos aquellos grupos sociales que muestran una mayor afinidad cultural y económica con la sociedad nacional que los Ese Eja. La categoría incluye por tanto no solo a agentes del estado, soldados y misioneros por ejemplo, sino también colonos, ribereños y hasta ciertos grupos indígenas "aculturados". Es decir, la categoría de deja es una categoría de 'alteridad' social, y, al igual que la categoría de etiikiana ("antiguo"), un punto de referencia a través del cual los Ese Eja construyen su propia identidad (cf. Gow, 1993). Los Ese Eja consideran que aquellos Ese Eja más familiarizados con la sociedad nacional son deja nisho ("gente falsa" o "parecido a gente"). 
declive en el numero de eyámikekwa y de "jefes", etii así como la mayor participación en actividades extractivistas y agrícolas ${ }^{14}$. Para los Ese Eja, cl ayahuasca, así como muchas otras plantas asociadas directa o indirectamente al contacto con deja, son dejaha ("de gente"), a diferencia de aquellas plantas identificadas como etiikianaha ("de los antiguos"). Es decir, los Ese Eja proyectan categorías de identidad social sobre los recursos vegetales circundantes.

Las justificaciones comúnmente expresadas para tomar ayahuasca son "para ver" y "para no enfermar". Las visiones inducidas por el ayahuasca y su interpretación simbólica constituyen el foco principal de la experiencia. A través de las visiones y los cantos, proyecciones del eshawa ${ }^{15}$ de la persona, los Ese Eja acceden e interceden dentro de una realidad, la realidad de los eshawa, normalmente inaccesible.

La raíz del verbo $[b a$ ]' significa "ver", "saber", y “conocer". Es decir, para los Ese Eja, la visión está íntimamente relacionada al conocimiento. El negativo de saber se articula siempre usando la forma condicional, ekweya bajima, "de-mi-saber-no-todavia", es decir, " no he visto / no conozco / no sé todavía." Las visiones del ayahuasca al igual que las de los sueños, articulan lo invisible a lo visible, lo desconocido a lo conocido, el conocimiento al poder ${ }^{16}$. La experiencia visionaria del ayahuasca, comparada al "cine", ofrece claves sobre aspectos críticos del universo, así como acceso y cierto control sobre fuerzas y eventos ordinariamente invisibles $y$, por tanto, usualmente fuera del control del individuo.

14 En otro trabajo (Alexiades, 1999) sugiero que este periodo de tiempo marcó una serie de cambios importantes en la etnobotánica Ese Eja, incluyendo la incorporación de nuevas técnicas y especies de cultivo así como de conocimientos sobre plantas medicinales. El concepto de eshawa es complejo, polivalente y de importancia central en la cosmovisión Ese Eja. Traducido por los Ese Eja al Castellano como 'alma', este corresponde a la fuerza vital de todo ser viviente, antropomórfico y ordinariamente invisible. Para los Ese Eja, todos los seres son manifestaciones de eshawa.

Ver Chaumeil (1983) para un excelente análisis de la relación entre la visión, el conocimiento y el poder en el chamanismo Yagua. 
"... he visto boa, se me acercaba, me queria hacer asustar... de alli, he visto una casa de concreto, bien hecha, bonita, con su chapa y su llave colgando, grande..."

En este ejemplo vemos dos símbolos o imágenes que se repiten con frecuencia; reptiles o animales acuáticos, y objetos manufacturados. Estas visiones son interpretadas de acuerdo a un código cultural. Casas, aviones, barcos, y automóviles aparecen en las visiones de ayahuasca como manifestaciones de los eshawa de varias especies, generalmente de árboles. Así por ejemplo, la casa de concreto corresponde a la casa del eshawa de mawi, el árbol shihuahuaco (Dipteryx spp., Fabaceae). El eshawa del árbol de la castaña, shiwi jaja (Bertholletia excelsa Bonpl., Lecythidaceae), se presenta como una lancha grande, provista de una larga chimenea.

Los Ese Eja cuentan que durante la toma de ayahuasca los participantes deben cantar "para que los eshawa (de las plantas y animales) no te lleven". El "dejarse llevar" tiene dos significados sobrepuestos. Por un lado, es sinónimo de "alocarse" (ñine), o sea, perder el control de sí mismo y/o de convulsionar. A otro nivel, "dejarse llevar" se refiere al acto de jeshékahé, el robo del eshawa de la persona por el eshawa de ciertos árboles:

"...el alma [eshawa] del palo te recoge, te lleva a su lado y te tiene allí castigado..."

El canto, como proyección del eshawa de la persona, protege al individuo de las agresiones de los eshawa. A través de las visiones, un individuo también puede "ver" y "actuar" sobre el eshawa de aquellos enfermos cuyos eshawa han sido capturadas por el árbol:

"...cuando toma ayahuasca le mira su alma (del enfermo) que se presenta, que está a su lado del palo, castigado. Alli le va a recoger (el ayahuasquero), el árbol le mezquina (kia nawe) pero (el ayahuasquero) le recoge a la fuerza, y la persona (que estaba enferma), se amejora..." 
Los actos, efectuados mediante la visión, se desarrollan al nivel de los eshawa, por lo cual no hay ningún tipo de contacto entre el ayahuasquero y el paciente. Es más, en muchos casos, y a diferencia de las sesiones de ayahuasca entre ribereños y otros grupos, los pacientes ni siquiera están presentes. En el siguiente relato, una mujer comenta sobre la visión de la noche anterior, en la cual se presenta un niño que había estado enfermo durante varias semanas:

"... Le hemos visto... ya estaba en kweyhana ${ }^{I 7}$... de alli le hemos llamado y se ha venido. Al día siguiente ya estaba mejor..."

Al recuperar el eshawa del niño del espacio de la muerte, los ayahuasqueros en este caso efectúan la reincorporación de la persona al mundo de los vivos, en efecto logrando su recuperación.

Al comienzo de la sesión de ayahuasca y durante los cantos, los participantes también le piden al ayahuasca "espantar" a los eshawa de enfermedades como kiyo ("fiebre"), oho ("tos"), see-see ("diarrea"), y wo'o ("viruela”). Para los Ese Eja, estas enfermedades infecciosas son los síntomas de los ataques de eshawa que viven "en las cabeceras" o "muy lejos" (kia wesha nei nei) y que ocasionalmente "atacan" a las comunidades Ese Eja. Al igual que los remedios vegetales y las medicinas "espantan" al eshawa patógeno del cuerpo de la persona, el ayahuasca "espanta" a los eshawa que invaden a la comunidad. Es decir, los actos aislados de enfermedad son vistos como la consecuencia de una agresión a nivel individual, mientras que los brotes de enfermedades infecciosas son vistos como el producto de una agresión a nivel social. El ayahuasca y los remedios vegetales tienen, por tanto, desde una perspectiva Ese Eja, efectos similares pero a escalas diferentes (Alexiades, 1999).

17 Kweyhana es el río o (en algunas versiones) carretera por el cual los muertos viajan hacia su destino al mundo de los muertos, también llamado kweyhana. 


\section{HISTORIA, CHAMANISMO Y RELACIONES CON LA NATURA- LEZA}

$\mathrm{Al}$ igual que muchas otras culturas amazónicas, la cosmovisión Ese Eja presupone la existencia de varios niveles y espacios, organizados tanto vertical como horizontalmente. Meshi (tierra), meshi dojó ("tierra-centro/ médula"), eya (cielo), los ríos, cabeceras y kweyhana, son algunos de los "espacios" que forman el universo en el cual la sociedad Ese Eja se haya enraizada (Chavarría, 1993). Estos "niveles" o "espacios" están a su vez poblados de seres humanos y sobrehumanos, cuyas necesidades, quehaceres y organización interna se asemeja a la de los Ese Eja. El hecho que estos "espacios sociales" estén separados entre sí a lo largo de un eje de tiempoespacio (ambos relacionados), no quiere decir que por ello existan de forma aislada o mutuamente independiente. En los ejemplos anteriores hemos visto como, por ejemplo, la caza, el eshasha-powi, la muerte, las enfermedades y las curaciones por parte del eyámikekwa constituyen formas de intercambio entre los Ese Eja y los edósikiana.

Al igual que los edósikiana controlan el acceso a la carne de monte, los deja controlan el acceso a otro recurso crítico: los objetos manufacturados, como son herramientas, cuchillos, motores y ropa. Al igual que los edósikiana "mezquinan", kia nawe, los animales, los deja "mezquinan" los objetos manufacturados. El adjetivo kia mase, que podría traducirse como agresivo, bravo y vindicativo, es también utilizado por los Ese Eja para describir las acciones de los edósikiana y los deja. En las secciones anteriores he sugerido que si bien el eyámikekwa tiene como rol articular la sociedad Ese Eja ante los edósikiana, los ancestros y a los animales del bosque, el rol del ayahuasquero está más vinculado a los deja, así como a ciertas plantas, árboles específicamente, y animales acuáticos. En esta sección exploraré en más detalle este modelo. Sugiero que el chamanismo eyámikekwa y el ayahuasca reflejan no solamente diferentes aspectos de la relación de los Ese Eja con su medio, sino que incorporan y expresan la experiencia histórica de dislocación, migración y de cambios en los patrones de asentamiento y subsistencia. 
La ubicación de los Ese Eja en la zona de transición entre el piedemonte andino y las zonas bajas de bosque tropical es un aspecto crítico de su entorno y del proceso de contacto con la sociedad nacional. Las circunstancias del proceso de colonización en la región durante los siglos XIX y XX favorecieron el aislamiento de las cabeceras y el poblamiento de las zonas bajas, ya que la economía extractivista y la colonización de la región fue propulsada por el caucho, jebe, y posteriormente la castaña, todos ellos recursos vegetales de las zonas bajas. La creciente articulación entre los Ese Eja y la sociedad nacional en este siglo supuso, por tanto, un desplazamiento desde el piedemonte hacia la planicie amazónica, ubicándose así más cerca a los centros urbanos. La experiencia histórica de contacto con la sociedad nacional y de migración río abajo imbuye el espacio con el tiempo y la memoria. Las memorias del pasado, yawajo nei nei, "hace mucho tiempo", funden el pasado histórico con el pasado mítico en el espacio de las cabeceras. El movimiento a través del espacio, río abajo, implica también el movimiento a través del tiempo, desde el pasado histórico-mítico al presente.

La migración hacia las partes bajas de los ríos y el contacto con deja, también alteró profundamente la relación entre los Ese Eja y su medio natural. Por un lado, la fauna, y sobre todo la flora ${ }^{18}$, son diferentes en las zonas bajas. La planta del ayahuasca por ejemplo, no se halla en la zona del piedemonte, siendo típica de los bosques bajos. La dislocación río abajo sobrepone una experiencia social, la mayor articulación con deja, a una experiencia ecológica, un mayor contacto con los recursos biológicos de las zonas bajas de los ríos.

La migración río abajo y el contacto con la sociedad nacional también supuso numerosos cambios en los patrones de subsistencia y manejo de recursos. Durante el periodo directamente anterior a su incorporación a

\footnotetext{
18 Desde el punto de vista de subsistencia Ese Eja, las diferencias en la flora son más notables que de fauna, ya que casi todas las especies principales de caza se encuentran en el piedmonte y en las zonas bajas.
} 
la economía de mercado los Ese Eja vivían en asentamientos dispersos y relativamente móviles, desplazándose de acuerdo a la distribución de los recursos naturales, y sobre todo de fauna. El plátano, complemento ideal del cazador-recolector seminómada, era el cultígeno principa ${ }^{19}$. A diferencia del pasado, centrado en las cabeceras y en la caza, recolección y cultivo del plátano, el contacto con deja ha implicado no sólo la migración y el asentamiento en los cursos bajos de los ríos, pero también un cambio en las actividades productivas, en las cuales la agricultura y la extracción de productos forestales comerciales han cobrado mayor importancia.

Los últimos cien años también han supuesto un cambio dramático en el tipo e intensidad de relaciones entre los Ese Eja y deja. Si bien los etiikiana tenían escaso contacto con deja, los contactos ocasionales eran caracterizados por intercambios "negativos", basados en predación y secuestros recíprocos:

“... [deja] tenia perro grande para comer Ese Eja, ese perro sabia matar Ese Eja, perro le seguía y con ecowiji (escopeta) [deja] le mataba. Asi era antes, mi abuelo me ha avisado. Acá hay harto Ese Eja que deja ha matado, en la playa enterrado, en cabeceras también hay harto lo que gente (deja) ha matado. Deja venia a matar Ese Eja. Epona (mujer) si no ha matado, le ha llevado a Bolivia, Riberalta, para que sea su mujer. También habia harto Ese Eja que ha matado a deja, porque etiikiana ("los antiguos") no conocía gente... recién cuando han bajado a la boca han conocido ese deja. Primero antiguo era miedoso de gente. Cuando escuchaba motor se esconde, hombre no mas se paraba... cuando trabajaba con padre ya no, él le ha enseñado. Deja mataba Ese Eja con perro, por eso nosotros pensamos cuando va a hacer así... deja que no conoce Ese Eja de repente va a hacer así..."

19 El plátano es muy rico en calorías y requiere relativamente poca inversión de trabajo para su cultivo, por lo cual es idóneo para cazadores-recolectores seminómadas. Cabe notar que la especie fue introducida a las Américas en el siglo XVI (Strover y Simmonds, 1987), extendiéndose rápidamente por toda la Amazonia. Este hecho es particularmente interesante dada la importancia central del plátano en la cultura y subsistencia Ese Eja a comienzos de siglo. 
La narrativa de esta mujer Ese Eja revela varios aspectos importantes de la relación Ese Eja-deja. Por un lado, la narradora identifica un cambio en el patrón de relaciones de intercambios con deja. El proceso de familiarización es un proceso también de socialización que implica el abandono de las relaciones de "predación" entre Ese Eja y deja. Sin embargo, la ultima frase expresa el temor que algún día se renueven las relaciones de predación. El temor de futuras agresiones por parte de deja se basa en otro aspecto característico de deja, desde el punto de vista Ese Eja: el hecho que deja-al igual que el edósikiana- es kia mase, agresivo, vindictivo y potencialmente peligroso. Incluso hoy día, las relaciones con deja mantienen un matiz de "predación", si bien a un nivel social y económico. Ciertamente, la relación entre Ese Eja y deja, al igual que entre el eyámikekwa y el edósikiana, es compleja y ambivalente. Por un lado hay un elemento de dependencia, cuya más clara expresión son las necesidades de objetos manufacturados, lo cual se traduce en la necesidad, y el deseo de entablar relaciones de intercambio con deja. A la vez, este contacto implica ciertos riesgos, los cuales emergen de dos cualidades deja: kia deja tai y kia mase. Las tensiones y conflictos con deja se expresan a su vez en actos de agresión física y, mas comúnmente, patógena, como son la brujería, y "daño de gente", por ejemplo.

Para resumir, la experiencia de contacto con deja y el desarrollo de relaciones con el "espacio" de la sociedad nacional ha implicado una dislocación hacia la boca de los ríos, un mayor énfasis en la agricultura y la interacción con los recursos vegetales. El movimiento a través del espacio y del tiempo ha supuesto un cambio en las dinámicas de intercambio entre los Ese Eja y su entorno. Las cabeceras, asociadas al pasado y a un estilo de vida seminómada centrada en torno a la caza, el cultivo del plátano, y las relaciones chamánicas con el edósikiana, se contraponen al presente. El presente está asociado a las partes bajas de los ríos, un estilo de vida más sedentario determinado a su vez por la distribución y disponibilidad de recursos controlados por deja. Los patrones de subsistencia del presente están más articulados a la economía de mercado, y por tanto centrados en la agricultura, incluyendo cultígenos como el arroz, y la extracción forestal (ver Figura 1). 
El chamanismo del eyámikekwa tiene como punto de referencia central aquellos elementos de la realidad Ese Eja asociados al pasado: netamente la caza, los emánokwana y las relaciones antagonistas (predatorias) con los deja. Los rituales de eshasha-poi por ejemplo, no pueden llevarse a cabo cuando hay deja en la comunidad, y todo aquello relacionado a los deja, incluyendo el idioma castellano, la luz artificial o animales como los gatos, "aterrorizan" a los emánokwana impidiendo la comunicación de los Ese Eja con sus ancestros. Los rituales del eyámikekwa suponen efectivamente un reencuentro momentáneo del presente y el pasado, yawaho nei nei, que excluye la incorporación de elementos deja.

Si bien el ayahuasca comparte con el eyámikekwa una función mediadora frente al mundo de los eshawa, a diferencia del chamanismo de eyámikekwa, el ayahuasca surge a raíz del contacto e interacción con deja. Ya he subrayado que los Ese Eja identifican el origen del ayahuasca al contacto con deja, y el hecho que, a diferencia de los rituales del eyámikekwa, los rituales del ayahuasca están empapados de imágenes y simbología de contacto con deja. Es más, a diferencia de los rituales del eyámikekwa, los ayahuasqueros cantan en un idioma que efectivamente incorpora y combina elementos Ese Eja con elementos castellanos.

En las narrativas sobre el eyámikekwa y el ayahuasca, los Ese Eja subrayan la diferencia entre ambos:

“...el ayahuasquero sabe llamar a las plantas, el eyámikekwa llama a los animales...tigre, masheke (lobo de río), be'o (buitre). El ayahuasquero casi no trabaja con los animales, mas con las plantas..."

"...con jono (ayahuasca), no viene huangana, viene con eshasha-powi"

Además de las diferencias en su relación simbólica con las plantas y animales, el eyámikekwa y el ayahuasca tratan nosologías y utilizan prácticas diferentes: 
“... eyámikekwa no usaba plantas, claro usaban hojas, así como para picadura de víbora... tabaco si usaban, introducian humo por hueso de guacamayo en la nariz para que seas mitayero (cazador)... pero el edósikiana no enseña las plantas, enseña a sacar la picada (chonteado, kekwa-kani) de plantas... la soga (ayahuasca) enseña a sacar mal aire, susto...enseña las plantas"

Si bien el eyámikekwa y el ayahuasquero emplean como marco de referencia la imagen de agresión patógena al nivel de eshawa, cada uno actúa sobre distintas formas de agresión. El eyámikekwa, mediante su relación con el edósikiana, cura aquellas enfermedades también asociadas al edósikiana, las cuales se clasifican principalmente bajo la categoría kekwákaní, chonteado, a su vez asociado simbólicamente a la imagen de la caza, y a los patrones de subsistencia y forma de vida ancestral en las cabeceras. El ayahuasquero, a través de jono, cura aquellas enfermedades asociadas a lo que los Ese Eja traducen como "cutipado" y "daño de gente". El cutipado tanto por árboles (jeshé-kaní) como por reptiles y ciertos pescados sin piel (jemí-kaní) está simbólicamente asociado al rapto, en el caso de jemi-kaní, habiendo, además, un matiz sexual ${ }^{20}$. Ciertamente, las imágenes del secuestro y de la apropiación sexual resuenan con una imagen central en la experiencia histórica de los intercambios "negativos” entre deja y Ese Eja: el rapto de mujeres y niños. Por último, y tal como indica el nombre, el daño de gente, un tipo de brujería, es una nosología también asociada al contacto con deja y no tratable por el eyámikekwa.

La posibilidad que jemikani y jeshé-kahé manifiesten elementos de contacto con deja, a través de la relación con plantas y ciertos animales, parece aún más probable cuando consideramos que las mujeres y los niños son precisamente los más susceptibles a este tipo de "rapto" por parte de los eshawa de los árboles y ciertos animales. Que ciertos arboles y animales sirvan, dentro del idioma chamánico, como símbolos de contacto con deja es ciertamente una posibilidad intrigante. De hecho, ya he mencionado

$20 \quad$ Jemi-kaní puede ser tratado por el eyámikekwa y el ayahuasca. 
como los Ese Eja proyectan etiquetas de identidad social sobre las plantas. El ayahuasca y otras plantas introducidas y/o de las zonas bajas son dejaha, "de gente". Una gran parte de las plantas comúnmente presentes en la visiones del ayahuasca son árboles de las zonas bajas. Incluso la referencia al agua y al mundo acuático puede tener significaciones de contacto con deja, ya que el descenso de las cabeceras también ha implicado un mayor contacto con el mundo del agua. Los ríos en las partes bajas son más anchos, y especies de zúngaro, hai, son típicos de los cursos bajos del río.

\section{CONCLUSIONES}

En los últimos 100 años, periodo latente en la memoria colectiva Ese Eja, se han dado una serie de procesos paralelos, todos ellos relacionados a la intensificación en las relaciones con el estado y sus agentes. El colapso demográfico, la migración, los cambios en los patrones de asentamiento y de subsistencia han contribuido a una mayor dependencia en la economía de mercado y a la reestructuración de ciertas instituciones sociales. La extinción inminente del eyámikekwa y la incorporación simultánea del ayahuasca, reflejan cambios importantes de como los Ese Eja perciben y articulan sus relaciones con medio social y ecológico. Sugiero que a través del ayahuasca, los Ese Eja expresan y manipulan nociones contemporáneas acerca de su identidad, la cual incluye relaciones esenciales pero frágiles y ambivalentes con un nuevo ambiente ecológico y social, caracterizado por los eshawa de árboles y de animales acuáticos, así como de deja.

El autodiagnóstico Ese Eja de la desaparición de los eyámikekwa refleja un proceso dinámico de cambio socioecológico y de identidad. La pérdida del contacto con los emánokwana y la desaparición de las manadas de huanganas constituyen metáforas que expresan la reorganización de la relaciones ecológicas. Al igual que los patrones de subsistencia y las relaciones económicas se han transformado, reforzando el contacto con deja y con plantas y disminuyendo el contacto con los animales y las cabeceras, tam- 
bién han cambiado los esquemas cosmológicos y la relación con los eshawa. La transformación del edósikiana después de su muerte, relatada por un ayahuasquero Ese Eja refleja la transformación y vitalidad del chamanismo Ese Eja:

"el edósikiana viejito, venia a tomar eshasha-powi para curar... cuando muere ese edósikiana viejo, nace [la planta del] ayahuasca..."

\section{AGRADECIMIENTOS}

Estoy profundamente agradecido a los Ese Eja por su hospitalidad y por sus extensas enseñanzas. Las ideas presentadas en este articulo se han desarrollado en gran parte gracias a mis conversaciones con David y Rafael Biaeja, Benjamín y Gregorio Huajo-Huajo, Roberto, Antonio y Rafael Kioshe, Ruperto Mamío, Roberto Masías, José Mishaja, Antenor Monje, Raquel Pino, Sixto Piquichewe, Emilia y Zacarías Sonihua, y Guido Tirina.

Agradezco a la Fundación Interamericana, Garden Club of America, Edward John Noble Foundation, Lawrence-Conoco-Dupont Fund, y el Instituto de Botánica Económica del Jardín Botánico de Nueva York por su apoyo financiero.

La Federación Nativa del Río Madre de Dios y sus Afluentes (FENAMAD), la Central Indígena de la Región Amazónica Boliviana (CIRABO), así como al Instituto Nacional de Recursos Naturales (INRENA, Puerto Maldonado y Lima) y al Museo de Etnografía y Folklore (MUSEF, La Paz) proporcionaron los debidos avales y permisos de investigación correspondientes. El INRENA, así como CESVI y la Fundación Pro-Naturaleza brindaron apoyo logístico crítico.

Mi ensayo refleja extensas y productivas conversaciones acerca de los Ese Eja, la región y el chamanismo con Garreth Burr, María Chavarría, Todd y Tammy Comstock, Alfredo García, Heinrich Helberg, Didier Lacaze, 
Tomás Moore, Daniela Peluso, y Mike y Chela Riepma. Garreth Burr, Robin Goodman, Daniela Peluso y Steven Rubinstein brindaron valiosos comentarios sobre una versión preliminar de este artículo. Agradezco a mis padres, Demetrio y Regine Alexiades por su ayuda editorial.

\section{BIBLIOGRAFÍA}

ALBERT, B.

1993 L'Or cannibale et la chute du ciel. Une critique chamanique de l'économie politique de la nature (Yanomami, Brésil). L'Homme 126128(2-4): 349-378.

ALEXIADES, M. N.

1999 Ethnobotany of the Ese Eja: Plants, Health and Change in an Amazonian Society. Tesis doctoral. New York: City University of New York.

BROWN, M. F.

1988 Shamanism and its discontents. Medical Anthropology Quarterly 2(2):102-120.

BURR, G.

1997 Eshawa! Vision, Voice and Mythic Narrative: An ethnographic presentation of Ese-eja mythopoeia. Tesis doctoral. Oxford: Magdalen College.

CHAUMEIL, J. P.

1983 Voir, Savoir, Pouvoir. Le chamanisme chez les Yagua du NordEst Péruvien. Paris: Editions de l'Ecole des Hautes Etudes en Sciences Sociales.

1985 Echange d'énergie: guerre, identité et reproduction sociale chez les Yagua de l'Amazonie Péruvienne. Journal de la Société des Américanistes 71:143-157. 
CHAVARRÍA M., M.C.

1993 Aspectos de la deixis espacial Ese Eja y su traducción al Español. Amazonía Peruana 22(23):89-106.

1996 Identidad y Armonía en la Tradición Oral Ese Eja (Tacana). Tesis doctoral. University of Minnesota.

CHAVARRÍA M., M.C. y F. Sánchez S.

1991 Ejatojabatiji: Buscando la somba de nosotros mismos. Aproximación etnográfica a la cultura Ese Eja. Manuscrito.

Dole, G.

1964 Shamanism and political control among the Kuikuru. Beitrage zur Volkerkunde Sud-Amerikas. Hanover, Germany: Festgabe fur Herbert Baldus.

GOW, P.

1993 Gringos and wild indians. Images of history in Western Amazonian cultures. L'Homme 126-128(2-4):327-347.

1994. River People: Shamanism and History in Western Amazonia. En: Shamanism, History and the State. Ann Arbor, MI: The University of Michigan Press.

HARNER, M. J.

1984 The Jívaro. People of the Sacred Waterfalls. Berkeley; University of California Press.

KIMURA, H.

1981 La mitología de los Esse Ejja del Oriente Boliviano. El dueño imaginario de los animales silvestres. Antropología. 2(3):1-22. 
POLLOCK, D. K.

1992 Culina shamanism: gender, power, and knowledge. En: Portals of Power: Shamanism in South America. E. J. M. Langdon y G. Baer, eds. Albuquerque: University of New Mexico Press.

REICHEL-DOLMATOFF, G.

1971 Amazonian Cosmos. The Sexual and Religious Symbolism of the Tukano Indians. Chicago: The University of Chicago Press.

STROVER, R. H. y N. W. SIMMONDS.

1987 Bananas. $3^{\circ}$ edición. New York: Longman Scientific and Technical / John Wiley and Sons. 